\title{
O.S.P.
}

L'orientation scolaire et professionnelle

$39 / 4 \mid 2010$

varia

\section{Clot, Y. Travail et pouvoir d'agir}

Paris : PUF

Jean-Yves Bonnefond

\section{(2) OpenEdition}

Journals

Édition électronique

URL : https://journals.openedition.org/osp/2959

DOI : 10.4000/osp.2959

ISSN : 2104-3795

Éditeur

Institut national d'étude du travail et d'orientation professionnelle (INETOP)

Édition imprimée

Date de publication : 31 décembre 2010

Pagination : 531-532

ISSN : 0249-6739

Référence électronique

Jean-Yves Bonnefond, «Clot, Y. Travail et pouvoir d'agir ", L'orientation scolaire et professionnelle [En ligne], 39/4 | 2010, mis en ligne le 31 décembre 2010, consulté le 28 juin 2022. URL : http:// journals.openedition.org/osp/2959; DOI : https://doi.org/10.4000/osp.2959

Ce document a été généré automatiquement le 29 septembre 2020

(c) Tous droits réservés 


\section{Clot, Y. Travail et pouvoir d'agir}

Paris : PUF

Jean-Yves Bonnefond

\section{RÉFÉRENCE}

\section{Paris : PUF}

1 En introduction, Y. Clot pose plusieurs questions à sa discipline. Notamment celle des rapports entre émotion et cognition: «l'activité n'est cognitive ou émotive qu'au second degré, elle est d'abord mouvement d'appropriation d'un milieu de vie par le sujet, ou amputation de celui-ci » (p. 6).

2 À travers l'activité se développe ou pas le pouvoir d'agir du sujet, dans un mouvement d'alternance fonctionnelle entre le sens et efficience, «le premier, fruit de l'échange, est source d'énergie. La seconde, sortie de la technique ou offerte par elle est source d'économie " (p.17). Le genre professionnel, apport central de la clinique de l'activité est un instrument collectif décisif du pouvoir d'agir.

3 La première partie de l'ouvrage est consacrée aux auteurs de référence en clinique de l'activité.

4 Tout d'abord Alain Wisner - ergonome - pour qui « l'opérateur est le créateur répété de sa tâche... ». Dans cette vision du sujet au travail, l'activité est synonyme de santé. Lev Vygotski et la perspective historico-développementale vient ensuite. Ce dernier s'intéresse avant tout aux passages entre activité personnelle et activité sociale. D'abord source de l'activité individuelle, la situation sociale devient ressource pour cette activité individuelle. Dans ce mouvement des sources et des ressources, l'activité individuelle se développe dans l'activité sociale. C'est à cette condition que l'individu devient sujet psychologique.

5 Y. Clot mobilise aussi L. Le Guillant parmi les fondateurs de la psychopathologie du travail. Il reprend à son compte les concepts de ressentiment et de condition. Il fait ensuite référence à Tosquelles et à la psychothérapie institutionnelle comme voie à 
suivre pour la psychopathologie du travail. Pour Tosquelles, l'activité n'est pas seulement un objet d'étude, mais un instrument clinique décisif : la clinique est action et pas seulement tableau, clinicien et patients travaillent pour soigner l'institution.

6 Sur les rapports entre psychopathologie du travail et psychanalyse, les travaux de Nathalie Zaltzman sur le travail de la culture (Kulturarbeit) comme filiation transhistorique indépendante des avatars œdipiens de chaque histoire individuelle sont des ressources pour penser la fonction psychologique du travail. Enfin, I. Oddone qui a transmis à la clinique de l'activité des bases pour penser l'action en milieu de travail avec l'instruction au sosie.

La deuxième partie a pour titre « L'activité : problèmes et concepts ".

8 Pour Yves Clot, la psychopathologie du travail permet de repenser l'activité. À la lumière des travaux de Vygotski, le réel de l'activité n'est pas que le réalisé. C'est aussi les activités suspendues, contrariées, empêchées, voire les contre activités: elles doivent donc être admises dans l'analyse. L'activité est conceptualisée comme triadique, c'est-à-dire comme "simultanément dirigée vers son objet et vers les autres activités portant sur cet objet, ou encore d'autres activités du sujet». Elle est médiatisée par des instruments techniques et symboliques qui sont des produits sociaux.

conception de la santé mobilisée est issue de Canguilhem pour qui la maladie participe de la santé, Canguilhem distingue normalité et normativité.. La normalité renvoie aux défenses, à l'adaptation, à la fixation sur une seule norme de vie, elle est selon lui pathologique. La santé c'est être "plus que normal ", c'est pouvoir suivre des nouvelles formes de vie, c'est être capable de normativité : de création de normes. D'un certain point de vue, c'est pouvoir se passer des défenses. La clinique de l'activité vise cela en soutenant la revitalisation du genre professionnel instrument collectif de l'activité individuelle.

Les concepts de genre et de style sont ensuite présentés. Entre la tache et l'activité il existe un troisième terme: le genre professionnel. Ce terme désigne des obligations implicites que partagent ceux qui travaillent pour arriver à travailler elles sont historiquement et collectivement construites. Le style est la recréation du genre en situation, un usage singulier renouvelé des instruments techniques et psychologiques. Ce n'est pas un attribut psychologique personnel : le style affranchit le sujet du genre en renouvelant ce dernier. La création stylistique, qui suppose un maniement maitrisé du genre, est la source d'un développement du genre mais aussi du sujet.

11 Y. Clot présente l'histoire de la méthode des auto-confrontations croisées toute tournée vers la remise au travail du genre professionnel. Cette partie se conclut sur le rapport entre le collectif et l'individuel trop souvent opposé. Le collectif serait dans l'individu. "L'activité collective apparaît deux fois dans le développement de l'activité individuelle. D'abord source de l'activité individuelle, la vie collective devient ressource pour cette activité individuelle. Elle obéit à une migration fonctionnelle » (p. 146).

12 La troisième partie porte sur les relations entre action et connaissance, sur la méthodologie et les méthodes.

13 Pour Vygostki «c'est seulement en mouvement qu'un corps montre ce qu'il est ». Il faut, par conséquent, provoquer le développement pour pouvoir l'étudier : il n'est pas accessible par des méthodes directes d'observation. En provoquant le développement par les méthodes, il est possible d'en comprendre le fonctionnement et d'étudier les 
invariants du développement. Y. Clot revient sur les méthodes. D'abord, l'instruction au sosie est présentée comme moyen de développement du vécu. Il montre en quoi la méthodologie à l'œuvre avec la méthode d'instruction au sosie en constituant un contact social "artificiel» avec soi- même permet une réentrée dans l'action, une répétition sans répétition, la mise au travail de l'action dans une autre activité avec le sosie. "Prendre conscience ne consiste donc pas à retrouver un passé intact par la pensée mais plutôt à le revivre et à le faire revivre dans l'action présente, pour l'action présente " (p. 199). Ensuite, l'auto-confrontation croisée qui vise à organiser un conflit entre le «déjà dit " à vocation monologique et le "pas encore dit " à vocation dialogique. L'auteur, à partir d'analyses détaillées de séquences de dialogues, montre comment les «migrations fonctionnelles» sont au principe du développement de la pensée. Les objets de discours font vivre des objets de débats et inversement. Les dialogues sur l'action font vivre des questions de métier qui alimentent des dialogues sur l'action. "Aussi bien en ce qui concerne les gestes ou encore les affects, on peut mieux expliquer ainsi comment se produit du nouveau » (p. 247).

Alors que le genre était initialement synonyme de métier en clinique de l'activité, l'ouvrage se termine par la proposition d'un modèle psychologique et développemental du concept de métier constitué de quatre instances.

Deux d'entre elles sont du coté de l'activité :

- personnelle : le métier s'exerce dans l'activité d'un sujet singulier;

- interpersonnelle : il ne peut y avoir d'activité sans destinataire, un métierne se fait pas seul.

Deux instances sont du coté du « donné », de « l'instituté » :

- impersonnelle : le métier, c'est aussi la tâche prescrite, la fonction.... Il existe indépendamment des personnes, c'est psychologiquement important ;

- transpersonnelle : le genre professionnel, c'est-à-dire cette mémoire collective, répondant du collectif de l'activité individuelle.

17 Tout est lié et tout peut se délier, « un métier privé des ressources vitales du travail collectif interpersonnel, sans répondant transpersonnel peut dégénérer en face-à-face ravageur entre un exercice personnel solitaire et des injonctions impersonnelle factices. » (p. 260). En résumé, on peut donc affirmer que la clinique de l'activité - avec les méthodes qui lui sont propres - vise à remettre le métier en mouvement jusqu'à la transformation de la tâche. Pour cela il s'agit d'organiser des migrations fonctionnelles entre les quatre instances dans la vie des sujets. 